\title{
Telearbeit in ländlichen Regionen Brandenburgs für private Unternehmen und öffentliche Verwaltungen
}

\author{
Prof. Dr. Peter Gadow, Dipl.-Psych. Hans Könecke, Dipl. Kfm. (FH) Susanne Reimer
}

In Brandenburg ist in den vergangenen Jahren in vielen Landesteilen von der Deutschen Telekom AG ein modernes Glasfasernetz verlegt worden. Die Wartezeiten der Bürger Brandenburgs auf einen Telefonanschluß haben sich auf wenige Monate, ja sogar Wochen verringert. Auch in kleinen, ländlichen Gemeinden wäre es nun möglich, über einen ISDN-Anschluß das breite Angebot der modernen Datendienste zu nutzen. Auf der CEBIT 1997 wurden zudem Satellitenanlagen präsentiert, welche dem privaten, freiberuflichen oder kleingewerblichen Nutzer den Datentransfer mit hohen Übertragungsraten ermöglichen würden. Diese Satellitenanlagen werden aber erst in den nächsten Jahren zu Preisen von wenigen tausend Mark angeboten werden können. Mit der nunmehr vorhandenen Infrastruktur für den Datentransfer sowie leistungsfähiger Rechentechnik beim Anwender wäre es folglich möglich, die Arbeitsplätze der Mitarbeiter und den Standort eines Unternehmens oder einer öffentlichen Verwaltung räumlich zu trennen.

Jenseits des technisch Machbaren stellen sich nunmehr die folgenden drei Fragen zur zukünftigen Schaffung von Telearbeitsplätzen in Brandenburg:

1.) Wieweit ist eine Optimierung des Leistungsprozesses in Einzelwirtschaften zu erwarten?

2.) Welche psychischen und sozialen Langzeiteffekte sind bei den Beschäftigten insbesondere in Teleheimarbeit zu erwarten?

3.) Welche volkswirtschaftlichen Effekte lassen sich insbesondere in den schwach besiedelten ländlichen Regionen Brandenburgs erzielen?

Diesen drei Fragen ist Frau Reimer im Rahmen ihrer Arbeit nachgegangen (ausführlich hierzu Reimer 1997). Es zeigte sich rasch, daß hierzu noch kaum gesicherte wissenschaftliche Erkenntnisse vorliegen - ja es existiert noch gar keine präzise Definition des Begriffes Telearbeit. „Anhand von vier Faktoren läßt sich Telearbeit von der konventionellen Büroarbeit abgrenzen...: Arbeitsort, Arbeitszeit, Ausstattung des externen Arbeitsplatzes und Rechtsform des Arbeitsverhältnisses" (Reimer, 9). Die Ausstattung des externen Arbeitsplatzes mit Hardund Software hängt natürlich wesentlich davon ab, welche Arbeiten - Buchhaltung, CAD-Konstruktion, Textverarbeitung u. v. a. mehr - an diesem Arbeitsplatz erstellt werden sollen. Dementsprechend liegen auch die Kosten für die Einrichtung eines Telearbeitsplatzes zwischen 8 und 25 TDM. Der Arbeitsort kann in der eigenen Wohnung des Mitarbeiters oder in einem Satel- litenbiiro des Arbeitgebers liegen. Es könnte auch ein Nachbarschaftsbüro von mehreren Arbeitgebern gemeinsam oder von einem eigenständigen elektronischen Dienstleistungsunternehmen betrieben werden, Entsprechend unterschiedlich wären die Rechtsformen des Arbeitsverhältnisses möglich als festes Arbeitnehmerverhältnis, Heimarbeitsverhältnis, freie Mitarbeit auf Basis eines Werkvertrages oder selbstständige gewerbliche oder freiberufliche Tätigkeit (vgl. Reimer 10-15).

Welche Vorteile können nun Einzelwirtschaften erwarten, die, in einer der soeben skizzierten Formen, Telearbeitsplätze schaffen wollen? Gewinnorientierte Einzelwirtschaften werden vermutlich solche Investitionen vor allem dann tätigen, wenn zumindest eine oder zwei Bedingungen zutreffen:

A. Die Marktstellung des Unternehmens im Wettbewerb könnte verbessert werden.

B. Die Akquisition und Motivation qualifizierten Personals durch Telearbeit würde verbessert.

Hypothese A. wäre zunächst einmal durch empirische Studien zu prüfen. Bei der explorativen Auswertung der vorhandenen deutschsprachigen Literatur im Rahmen der Diplomarbeit von Reimer konnten hierzu keine Aussagen gefunden werden. Zu erwarten wäre eine verbesserte Marktstellung nach Ansicht der Autoren durch Telearbeit dann, wenn nennenswerte Kosteneinsparungen zu realisieren wären, die als Vorteil im Preiswettbewerb an die Kunden weitergegeben werden können. Bei der Telearbeit im Rahmen eines festen Arbeitsverhältnisses ist jedoch keine Reduzierung der Kosten zu erwarten, solange im Unternehmen selbst noch ein $\mathrm{Ar}$ beitsplatz für den Beschäftigten bereit gehalten werden muß (vgl. Reimer, 17ff). Interessant ist hierbei auch der Vergleich mit Japan, wo die Telearbeit vor allem in Form von Satellitenbiiros realisiert wurde. Teleheimarbeit dagegen ist in den dicht besiedelten Ballungszentren auf den japanischen Inseln kaum möglich - die verfügbare Wohnfläche je Einwohner ist dafür viel zu gering (vgl. Reimer, 34). Rechtlich gesehen sind viele der in den Satellitenbüros Beschäftigten selbstständige Gewerbetreibende, die durch Kontrakte an ihre Auftraggeber gebunden sind. Wieweit diese Form des „Outsourcing“ (Verlagung von Funktionen aus dem Unternehmen zu selbstständigen Lieferanten) zu einer Kostenentlastung - und somit zu einer verbesserten Marktstellung - auch bei deutschen Unternehmen führen könnte, ist nach unserem Wissen noch nicht empirisch untersucht worden. 
Die derzeit in Deutschland verfügbaren Studien zur Telearbeit beschäftigen sich vor allem mit der genannten Hypothese B. - den möglichen Effekten der Telearbeit im Rahmen der betrieblichen Personalpolitik. Mögliche Vorteile der Telearbeit werden gesehen insbesondere in einer besseren Verbindung von Arbeits- und Familienleben, einer flexibleren Arbeitszeitgestaltung und der Reduzierung der Fahrtzeiten.

Wieweit wäre nun zu erwarten, daß Einzelwirtschaften zukünftig Telearbeit als Mittel der Personalpolitik einsetzen werden? Zur Beantwortung dieser Frage erscheint es uns sinnvoll, nach den Brandenburger Wirtschaftzweigen zu differenzieren. Gleichzeitig wird hierbei diskutiert, welche volkswirtschaftlichen Effekte in den nächsten Jahren zu erwarten sind, sofern tatsächlich weitere Telarbeitsplätze geschaffen werden.

\section{Land und Forstwirtschaft}

Bei jeder „Landpartie“ jenseits des Autobahnringes um Berlin wird dem Auge des Betrachters offensichtlich, mit welch gravierenden Problemen die Land- und Forstwirtschaft seit 1990 zu kämpfen hat. Nur etwa 60.000 Erwerbstätige hat dieser Wirtschaftszweig heute noch (vgl. Reimer, 38). Die verbleibenden Unternehmen haben einen hohen Verwaltungsaufwand u.a. zur Abrechnung der EU-Beihilfen zu leisten. Eine Verlagerung dieser Tätigkeiten in Telearbeitsplätze ist jedoch nicht zu erwarten, da ja diese Verwaltungsarbeit bereits in den ländlichen Gemeinden geleistet wird.

\section{Ernährungswirtschaft}

Dies ist ein kleiner, aber wichtiger Teil der Brandenburger Wirtschaft. Erzeugnisse aus dem Spreewald oder Eberswalder Wurstwaren sind über die Landesgrenzen hinaus bekannt. Für die Telearbeit geeignete Tätigkeiten in der Forschung und Entwicklung, Konstruktion sowie allgemeinen Verwaltung haben jedoch in diesem Wirtschaftszweig einen vergleichsweise geringen Anteil an der Gesamtzahl der Arbeitsplätze.

\section{Verarbeitendes Gewerbe}

Zusammen mit der Ernährungswirtschaft hat Brandenburg in diesem Wirtschaftszweig insgesamt ca. 900 Unternehmen unterschiedlicher Größe. Untersuchungen in Nordrhein-Westfalen ergaben ein Potential von ca. $9 \%$ der Arbeitsplätze für die Telearbeit in diesem Wirtschaftszweig (vgl. Reimer, 31). Bei der derzeitigen Struktur dieses Wirtschaftszweiges in Brandenburg dürfte das Potential in Brandenburg jedoch weit geringer sein. Hier werden vor allem Halbwaren und Vorerzeugnisse wie Baustoffe, Stahl, Papier u.ä. Produkte in Massenfertigung hergestellt. Ein Potential fuir Telearbeit vermuten wir nur in den wenigen Dutzend Brandenburger Kleinund Mittelunternehmen der Elektrotechnik, Meß-, Steuer- und Regeltechnik sowie der Verkehrstechnik.

\section{Bauwirtschaft}

Bei der Erstellung von Angeboten sowie der Abrechnung der Bauleistung entsteht, im Vergleich zu den vorher genannten Branchen mit Massen- oder Serienfertigung, ein höherer Verwaltungsaufwand, da jeder einzelne Auftrag gesondert kalkuliert und abgerechnet werden muß. Die Kapital- und Qualifikationsstruktur der Klein- und Mittelunternehmen dieses Wirtschaftszweiges sowie die nachlassende Baukonjunktur stehen zur Zeit allerdings einer weiteren Verbreitung der Telearbeit entgegen. Es wäre detailliert zu prüfen, wieweit durch ein "Outsourcing" an Teleservicecenter die örtlichen Handwerksmeister verschiedener Gewerke spürbare Zeitoder Kostenersparnisse in ihrer Verwaltungsarbeit realisieren könnten.

\section{Groß- und Einzelhandel, Gastronomie und Hotelerie}

Der EDV-Einsatz ist bei mittleren und größeren Unternehmen des Handels wie auch der Hotelerie bereits weit verbreitet. Die Autoren sehen hier ein Potential insbesondere für die Teleheimarbeit einzelner kaufmännischer Angestellter der jeweiligen Unternehmen.

\section{Transport und Logistik}

Moderne Logistik ist ohne EDV-Einsatz schlichtweg nicht vorstellbar. Die Autoren regen an, gemeinsam mit den Kollegen der Studiengänge Logistik und Wirtschaftsinformatik sowie interessierten Brandenburger Unternehmen ein Modellprojekt zur Telearbeit in diesem Wirtschaftszweig zu initiieren, mit dem das tatsächliche Potential weiter evaluiert wird.

\section{Wirtschaftsnahe Dienstleistungen}

Die Bandbreite dieser Dienstleistungen reicht vom Facility Management über Consulting und Engeneering, Werbung, Übersetzerdienste bis hin zu den Leistungen der Notare und Wirtschaftsprüfer. Jede dieser Dienstleistungen erfordert eigentlich eine gesonderte, detaillierte Analyse ihres Potentials zur Telearbeit. Einige dieser Dienstleistungen erfordern die räumliche Nähe zu den Standorten größerer Unternehmen, deren Zahl jedoch in Brandenburg äußerst gering ist. Anderer dieser Dienstleistungen können standortunabhängig angeboten werden. Gerade diese Leistungen sind für Telearbeit prädestiniert, sie erfordern einzig eine gute Qualifikation der Mitarbeiter.

\section{Soziale Dienstleistungen privater Träger}

Vielfältige Aufgaben in den Bereichen Bildung, Gesundheit, Kultur u.a. mehr werden in der Bundesrepublik Deutschland von den Trägern der Freien Wohlfahrtpflege wahrgenommen. Einige dieser Dienstleistungen 
könnten durch den Einsatz moderner DV-Technik eine neue Qualität gewinnen. Zwei unter Strukturprämissen interessante Ideen behandelt Reimer in ihrer Arbeit. In den ländlichen Gemeinden Brandenburgs bleiben vor allem die älteren Menschen zurück. Hier wäre es möglich, durch sog. Telebetreuung (vgl. Reimer, 61ff) diesen Menschen gezielt lebenspraktische Ratschläge, Gesundheitstips und Anleitungen zu gymnastischen Übungen zu geben, ohne daß jeweils noch ein persönlicher Besuch durch Betreuungs- bzw. Pflegepersonal notwendig wäre. Vergessen werden soll aber an dieser Stelle nicht, daß bei vielen Erkrankungen ein guter persönlicher Kontakt zum Arzt resp. anderen Heilberufen nach wie vor die Basis des Therapieerfolges ist.

Zur Sicherung und Verbesserung des Bildungsangebotes im ländlichen Raum schlägt Reimer die Einrichtung von Telebibliotheken auch in kleineren Gemeinden vor (vgl. Reimer 67ff). Entsprechend qualifizierte Bibliothekare o.ä. Mitarbeiter erledigen für ihre Kunden die Literaturrecherche, Bestellung und Abrechnung, Ausgabe sowie Rücknahme der Bücher. Durch dieses Angebot könnte nach Ansicht der Autoren die Qualifikation der ländlichen Bevölkerung für Tätigkeiten in anderen Wirtschaftszweigen entscheidend gestützt werden.

\section{Dienstleistungen öffentlicher Haushalte}

Letztlich wollen wir kurz diskutieren, wieweit die Leistungen des Landes Brandenburg und seiner Dienststellen, bsw. die Universitäten und Fachhochschulen, verstärkt durch Teleheimarbeit der Mitarbeiter (Beamte oder Angestellte) erbracht werden könnten. Gleiches gilt für die Kommunen sowie die Sozialversicherungen. Neben der bekannt schwierigen Haushaltslage, welche eine rasche Anschaffung der notwendigen Hard- und Software derzeit bremst, steht dem auch das öffentliche Dienstrecht entgegen: In vielen Verwaltungen ist es den Mitarbeitern untersagt, Akten zur Bearbeitung mit nach Hause zu nehmen. Für dieses Problem scheint zumindest die LVA Hannover eine Lösung gefunden zu haben, welche insbesondere Frauen mit kleinen Kindern schon seit Jahren die Möglichkeit zur Teleheimarbeit gibt. Entsprechende Lösungen müssen auch für die Verwaltungen in Brandenburg gefunden werden, da die Verwaltung in diesem Bundesland nach Meinung der Autoren „eine Lanze für die Teleheimarbeit“ brechen muß.

Die folgende Darstellung enthält eine - noch empirisch zu erhärtende - Schätzung des Potentials fuir Telearbeit in den verschiedenen Wirtschaftszweigen Brandenburgs. Die privaten Unternehmen werden in den nächsten Jahren nur in wenigen Branchen in nennenswertem Umfang Telearbeitsplätze schaffen können, zumal kaum zu steuern ist, wieweit diese Arbeitsplätze in den ländlichen Regionen Brandenburgs entstehen. Um dort Arbeitsplätze zu schaffen, sollten nach Auffassung der Autoren die öffentlichen Verwaltungen mit gutem Beispiel bei der Schaffung von Telearbeitsplätzen in ländlichen Regionen vorangehen.
Wirtschaftszweig

Land und Forstwirtschaft

Ernährungswirtschaft

Verarbeitendes Gewerbe

Bauwirtschaft

Groß- und Einzelhandel,

Gastronomie und Hotelerie

Transport und Logistik

Potential fuir Telearbeit

wirtschaftsnahe Dienstleistungen

soziale Dienstleistungen privater Träger

Dienstleistungen öffentlicher Haushalte

$\begin{array}{ll}\text { Legende: } & \\ - & \text { Potential kaum vorhanden } \\ + & \text { geringes Potential } \\ ++ & \text { größeres Potential }\end{array}$

Bei der Ausschöpfung der hier geschätzten Potentiale gilt es ferner zu beachten, daß die genannten Tätigkeiten neben guten EDV-Anwenderkenntnisse auch in der Regel ein erhebliches Fachwissen verlangen. Dies entspricht nicht der aktuellen Qualifikationsstruktur der Bevölkerung in den ländlichen Regionen Brandenburgs (vgl. Reimer, 37f). Hier sind von Seiten der Wirtschaftsförderung und der Bundesanstalt für Arbeit noch erhebliche Anstregungen notwendig, um überhaupt Mitarbeiter bsw. für ein Tele-Servicecenter qualifizieren zu können. Als Kristallisationspunkt zur Verbesserung der Qualifikationen im ländlichen Raum könnten nach Auffassung der Autoren die bereits beschriebenen „Telebibliotheken" dienen, welche eng an die bereits bestehenden Kreisvolkshochschulen oder private Bildungsträger angebunden werden sollten, um dort den praktischen Unterricht durchzuführen. Um ein Zeichen für Modernität im ländlichen Raum zu setzen, sollten bald weitere Projekte realisiert werden. Derzeit gibt es nach dem Wissen der Autoren zwei Brandenburger Projekte in Golm und Strausberg, deren Wirkungen jedoch in den peripheren Landesteilen - bsw. der Prignitz oder der Uckermark - nicht mehr spürbar sein dürften. In den ländlichen Regionen sind zunächst einmal Qualifizierungsangebote für die Bevölkerung zu schaffen. Erst wenn Infrastruktur, Qualifikation und Motivation vorhanden sind, könnten darauf aufbauend Tele-ServiceCenter geschaffen werden, welche ihre Dienstleistungen der Brandenburger Wirtschaft anbieten. Ähnliche Pilotprojekte wurden bsw. in Bayern als „TeleService Fränkische Schweiz" von der Fraunhofer-Gesellschaft initiiert und von der EU gefördert (vgl. Reimer, 59f); derzeit aber ist dieses Projekt noch nicht kommerziell erfolgreich und unabhängigvon öffentlicher Förderung.

Neben der Finanzierung wird die weitere Verbreitung der Telearbeit in Brandenburg entscheidend davon abhängen, welche psychosozialen Effekte die einzelnen Mitarbeiter davon erhoffen oder befürchten. Mögliche positive Effekte - eine bessere Verbindung von Arbeitsund Familienleben, flexiblere Arbeitszeitgestaltung und Reduzierung der Fahrtzeiten - wurden bereits genannt. Ein offensichtlicher Nachteil ist dagegen die räumliche Isolation des Mitarbeiters von anderen Kollegen im Un- 
ternehmen. Leistungsverdichtung und -druck können unmittelbar ins Familienleben einwirken (vgl. Reimer, 13). Die stark differierende Qualität der im Rahmen von Telearbeit möglichen Tätigkeiten läßt zumindest bei einigen Tätigkeiten die Gefahr einer Monotonie erkennen. Die vorwiegend repetitive Ausfuihrung von Tätigkeiten der Telearbeit birgt ferner die Gefahr einer Stagnation des beruflichen Wissens, welche sich negativ auf die Karrierechancen des Mitarbeiters auswirkt.

Gegen die Einführung von Telearbeit sprechen ferner aus der Sicht der Unternehmen Probleme des Datenschutzes (vgl. BMWi, 9). Bei den erwarteten volkswirtschaftlichen Effekten hofft man zunächst einmal auf die Schaffung und Sicherung von Arbeitsplätzen durch die Telearbeit. Bei der differenzierten Betrachtung wird jedoch deutlich, daß durch Telearbeit vor allem erreicht werden kann eine Neuorganisation und räumliche Disaggregation der Tätigkeiten in Unternehmen und Verwaltungen. Eine räumliche Verlagerung von Arbeitsplätzen - wenn sie denn gewollt wird - in ländliche Regionen ist dadurch binnen weniger Wochen möglich, sofern hierfür das Personal entsprechend qualifiziert ist. Per Saldo positive Beschäftigungseffekte zum Abbau der Arbeitslosigkeit lassen sich dagegen nur erzielen, wenn in ländlichen Regionen DV-basierte Dienstleistungszentren entstehen, die ihre Leistungen erfolgreich regional und überregional vermarkten (vgl. Reimer, 73ff). Die zu erwartenden ökologischen Effekte (vgl. von Weizsäcker u. a.) dürften dagegen in den nächsten Jahren marginal sein. Sicherlich ist es möglich, in den nächsten Jahren in Brandenburg einige hundert Telearbeitsplätze zu schaffen, wodurch die Fahrten zwischen Arbeitsplatz und Wohnung entfallen würden. Einsparungen wie bei einer Kundenberaterin der Telekom AG Frankfurt/Oder „ich muß nicht mehr jeden Tag 140 km zur und von der Arbeit fahren" (zitiert in BMWi, 35) sind längst nicht immer in der genannten Höhe zu realisieren. Einsparungen bei den Fahrtzeiten allein dürften jedoch nicht dazu führen, daß diese Mitarbeiter auch auf die Anschaffung eines KfZ verzichten würden. Unter den derzeitigen Prämissen der Verkehrspolitik sichert nach wie vor ein KfZ die Mobilität der ländlichen Bevölkerung.

\section{Fazit}

Der wichtigste Effekt bei der Schaffung von Telearbeitsplätzen in ländlichen Regionen Brandenburgs liegt nach Meinung der Autoren eindeutig in der Stärkung der Attraktivität des ländlichen Raumes. Neben anderen Maßnahmen zur Strukturverbesserung sind dort rasche und konsequente Bemühungen zur Schaffung von Telearbeitsplätzen notwendig, um den dramatischen Bevölkerungsrückgang im ländlichen Raum durch das Angebot von qualifizierten Arbeitsplätzen in ausgewählten Wirtschaftszweigen und öffentlichen Verwaltungen zu stoppen, zumindest aber abzuschwächen. Unter den geschilderten Bedingungen sind diese gewünschten Erfolge sicherlich nur mittelfristig binnen 5 bis 8 Jahren zu erzielen.

\section{Liłeratur}

Bundesministerium für Wirtschaft BMWi (1996):

Telearbeit - Ein Ratgeber für Arbeitnehmer, Freiberufler und Unternehmen; Bonn

Hendricks, Bernd (1996):

Mein Büro ist zu Hause - Ihre Chancen in der neuen Welt der Telearbeit; Stuttgart

Reimer, Susanne (1997):

Telearbeit in ländlichen Regionen Brandenburgs für private Unternehmen und öffentliche Verwaltungen; Diplomarbeit an der TFH Wildau

Weizsäcker, Ulrich von u.a. (1996):

Faktor vier; München

\section{Autoren}

\section{Prof. Dr. Peter Gadow}

Dipl.-Psych. Hans Könecke

Technische Fachhochschule Wildau

Fachbereich Wirtschaft, Verwaltung und Recht

Tel. $\quad++49$ (0) $3375 / 508325$

Fax ++49 (0) $3375 / 508388$

Dipl. Kfm. (FH) Susanne Reimer

Tel. $\quad++49(0) 30 / 2478814$ 